\title{
Artificial Intelligence Based Model for Channel Status Prediction: A New Spectrum Sensing Technique for Cognitive Radio
}

\author{
Sandhya Pattanayak ${ }^{1}$, Palanaindavar Venkateswaran ${ }^{2}$, Rabindranath Nandi ${ }^{2}$ \\ ${ }^{1}$ Department of Electronics and Communication, Narula Institute of Technology, Kolkata, India \\ ${ }^{2}$ Department of Electronics and Communication, Jadavpur University, Kolkata, India \\ Email: sandhya.pattanayak@yahoo.com,pvwn@ieee.org,robnon@ieee.org
}

Received January 23, 2013; revised February 18, 2013; accepted March 13, 2013

\begin{abstract}
The recent phenomena of tremendous growth in wireless communication application urge increasing need of radio spectrum, albeit it being a precious but natural resource. The recent technology under development to overview the situation is the concept of Cognitive Radio (CR). Recently the Artificial Intelligence (AI) tools are being considered for the topic. AI is the core of the cognitive engine that examines the external and internal environment parameters that leads to some postulations for QoS improvement. In this article, we propose a new Artificial Neural Network (ANN) model for detection of a spectrum hole. The model is trained with some pertinent features over a channel like SNR, channel capacity, bandwidth efficiency etc. The channel capacity status could be identified in a quantized index form $\{0,1\}$. Some simulation results are presented.
\end{abstract}

Keywords: ANN; Cognitive Radio; Spectrum Sensing; Cognitive Engine

\section{Introduction}

The electromagnetic radio spectrum is a limited but precious natural resource. With the technological development of various wireless communication systems and demand for frequencies, there is a common notion of scarcity of spectrum at which these wireless devices operate [1]. Recent literature indicates that substantive portion of remains underutilized as shown in Figure 1 owing to the current fixed allocation policy, e.g. one observes in [2-4] that only $2 \%$ of licensed spectrum is being used in USA at a given time. Further reports show that the spectrum utilisation is only $22 \%$ in urban area and about $3 \%$ in rural area [2-8].

The cognitive radio is now being considered as an intelligent device that enables an efficient use of spectrum. To the OOPDAL Loop [9] a new feature knowledge base is added [10] to interact with the environment and store the processed information in the database to take decisions [10].

As per literature $[11,12]$ the various spectrum sensing methods are:

- Matched filter detection technique [13];

- Energy detection techniques [14,15];

- Cyclostationary feature detection technique $[16,17]$.

In this paper we propose an algorithm with artificial intelligence for spectrum sensing hence determine the occupancy status of a channel, thus enabling opportunistic spectrum access. Recently Artificial Intelligence (AI) based methods are also receiving significant research attention. It has been seen that AI forms the basic core of the cognitive engine that may be conveniently used for the improvement of certain QoS parameters of wireless communication system via cognizance. Literature survey further indicates that newer techniques based on ANN and genetic algorithms are also being investigated for $\mathrm{CR}$ spectrum sensing $[18,19]$.

We propose a new ANN model with a view to examine and subsequently predict the status of the channel in the TV band. This prediction algorithm enables one to decide whether a given band is occupied by a primary user (PU) or not - thus leading to an effective identification of a white space or spectrum hole for possible allocation to a secondary user (SU). In the proposed methodology the scenario considered is a CR-based scanning system placed within the range of a transmitting TV station. The occupancy status of the TV channel is determined based on certain pertinent channel parameters, like signal to noise ratio (SNR), channel capacity, bandwidth (BW) efficiency and the distance (d) of the scanning system from the primary TV base station. Our main aim is to determine the channel occupancy status in a quan- 


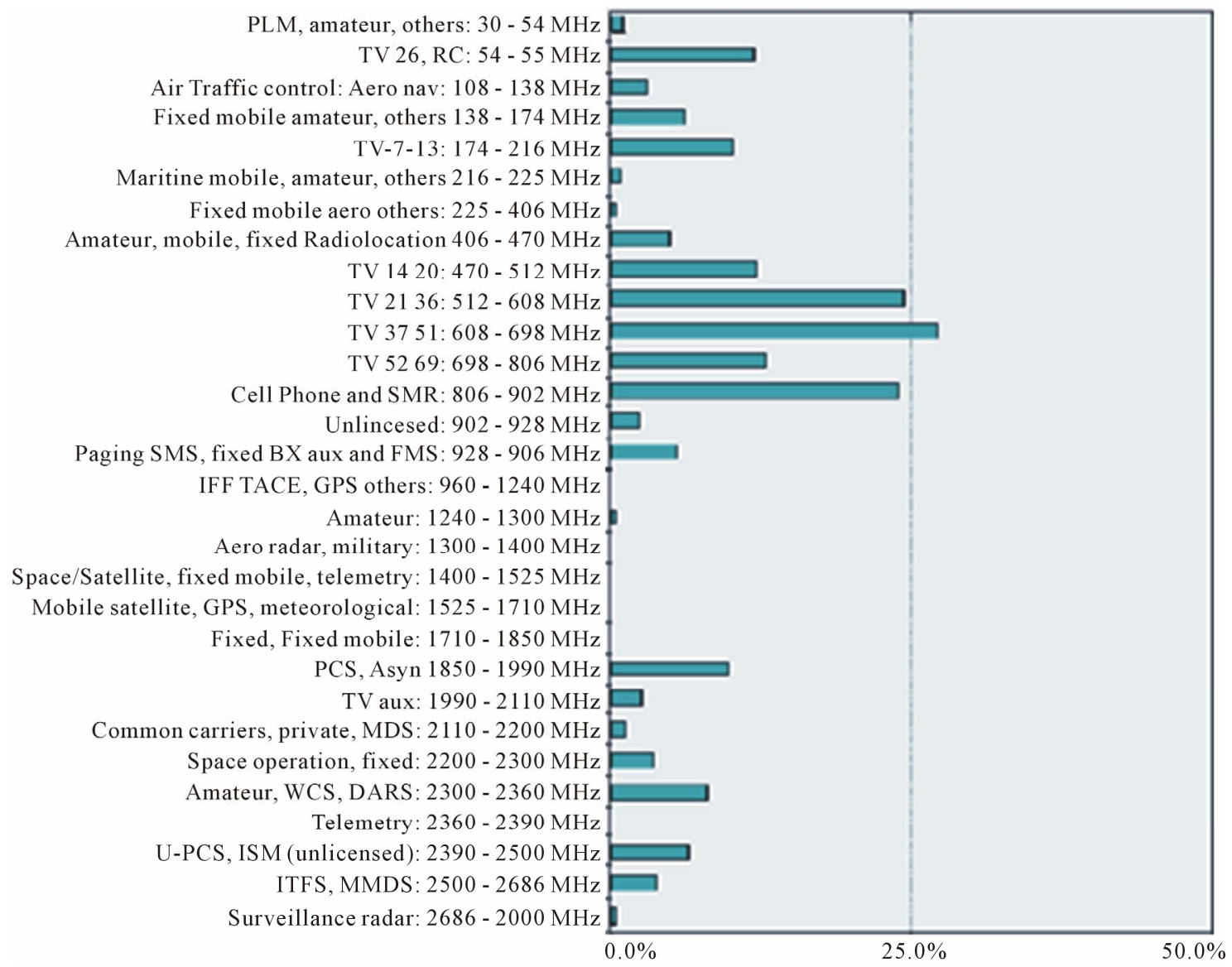

Figure 1. Spectrum utilization profile [4].

tized index $\{0,1\}$ after suitably formulating the algorithm with appropriate training of the ANN engine.

\section{Literature Overview}

\subsection{Spectrum Sensing}

Spectrum sensing is the capability of the cognitive radio to detect unused band and allot it to a secondary userOpportunistic Spectrum Access.

Up-to-date literature survey reports that the following basic signal processing techniques to determine the holes in the spectrum:

The ANN engine predicts the occupied channel as " 1 " and unoccupied channel as "0". Some Matlab based simulation results that compares favorably with theoretically calculated results are included.

\subsubsection{Energy Detection Technique [14,15]}

The detection of the primary signal is based on the hypothesis

$$
H_{0}: \eta=0 \text {, primary signal absent }
$$

$H_{1}: \eta=1$, primary signal present

where $\eta$ is 0 or 1 which determines the presence or absence of the primary signal respectively.

$$
\begin{gathered}
H_{0}: y(t)=n(t) \\
H_{1}: y(t)=h x(t)+n(t)
\end{gathered}
$$

where $x(t)$ is the primary user's signal to be detected, $n(t)$ is the additive white Gaussian noise, $h$ is the channel gain; $H_{0}$ is a null hypothesis indicating white space and $H_{1}$ indicates the channel is occupied with a primary signal. The detection is performed by taking the average energy level of the samples and compare with a threshold level

$$
T=\frac{1}{N} \sum_{t=1}^{N}|y(t)|^{2}
$$

where $T$ is the total energy observed over $N$ samples. A good detector has less probability of false alarm. In [2023], techniques to reduce the false alarm have been developed.

\subsubsection{Feature Detection Technique [16,17]}

The feature detection of the primary signal technique is also unique in itself as the signal transmitted by the primary user has unique features like a particular modulation rate, carrier frequency, central frequency and band- 
width [17,24]. Cyclostationary feature detection is applied in spectrum sensing as the transmitted signals are usually modulated signals coupled with sine wave carriers, pulse trains, hopping sequences, or cyclic prefixes. This technique distinguishes noise from primary signal, as additive noise is generally wide-sense stationary (WSS) with no correlation [25]. Hence the cyclostationary feature detection technique distinguishes different transmissions from the primary signals and noise.

\subsubsection{Matched Filter Detection Technique [13]}

If secondary users know information about a primary user's signal a priori, then the optimal detection method is the matched filtering [26]. Matched Filter detection technique is used for the detection of the primary user. Here the received signal is correlated with the already known signal in order to identify the primary user [27]. Hence a perfect knowledge of the signal like operating frequency, modulation technique etc is required to apply this technique.

\subsubsection{Statistical Covariance-Based Sensing $[28,29]$}

The Statistical Covariance-Based Sensing uses statistical covariance matrices of the received signal and noise to differentiate between them $[28,29]$.

\subsubsection{Learning/Reasoning-Based Sensing}

Recently learning based techniques like Hidden Markov process, ANN [30] and Genetic algorithm [31] are used for spectrum sensing.

\subsubsection{Cooperative Sensing Technique [32-34]}

The cooperative sensing techniques are used to reduce the probability of false alarm and improve spectrum sensing. As we know that spectrum sensing is limited by noise, uncertainty and fading resulting in low SNR of the primary user. Hence the secondary user will not be able to detect the primary user within its range leading to Hidden terminal problem of primary user and sensing speed [32-35]. Hence to improve spectrum sensing and remove hidden terminal problem, the concept of cooperative sensing has come up.

In centralized cooperative sensing a central controller collects information from the users and decides which band is vacant: whereas in distributed cooperative sensing the secondary users distribute information among themselves to decide the status of the band, hence a central controller is not required [32]. In this paper we consider ANN learning based sensing technique for determining the spectrum status algorithm.

\subsection{Spectrum Allocation}

Once the white spaces are detected the spectrum has to be allocated to the SU. This SU has to use the spectrum ef- ficiently and coexist with the PU.

The concept of open spectrum sharing is allowed in the ISM band and the sharing of the licensed spectrum among the primary and secondary user is referred as hierarchal spectrum sharing or licensed spectrum sharing [36].

Spectrum allocation and sharing schemes are categorized as spectrum underlay and spectrum overlay [36]. In the spectrum underlay the secondary users are allowed to transmit data in the licensed spectrum when the primary user is also transmitting, whereas spectrum overlay also referred to as Opportunistic spectrum access which allows the SU to transmit only when the PU is not transmitting over the band.

The second classification is based on the network architecture. The spectrum allocation is said to be centralized when a centralized controller controls the spectrum allocation. Spectrum allocation is said to be distributed when there is no centrally controlling station for spectrum allocation as in the distributed sensing the decision is taken on the basis of the local allocation.

\subsection{Spectrum Handoff [37]}

When the PU reclaims the channel provided to the $\mathrm{SU}$, the SU has to stop transmission of data over the channel and search for other unoccupied channel. This is the concept of spectrum handoff.

To perform these functions cognitive engine (CE) has been suggested to dynamically take decisions depending on the radio parameters.

The CE [38] consists of user interface, sensor interface, radio interface, scheduler and cognitive core as shown in Figure 2. The Cognitive core is the intelligent part of the $\mathrm{CE}$ consisting of the knowledge base, reasoner, learner and optimizer. The learning and optimization process is performed by the AI techniques and the knowledge base stores the experiences gained from the different scenarios [38]. The scheduler helps to learn, reason and optimize and the radio interface controls the radio parameters for optimized use of radio resources like bandwidth, spectrum, power, etc. Hence the radio has to be a reconfigurable radio. All the optimized outputs and the scenario details are stored in the database which will act as reference in future for similar configuration.

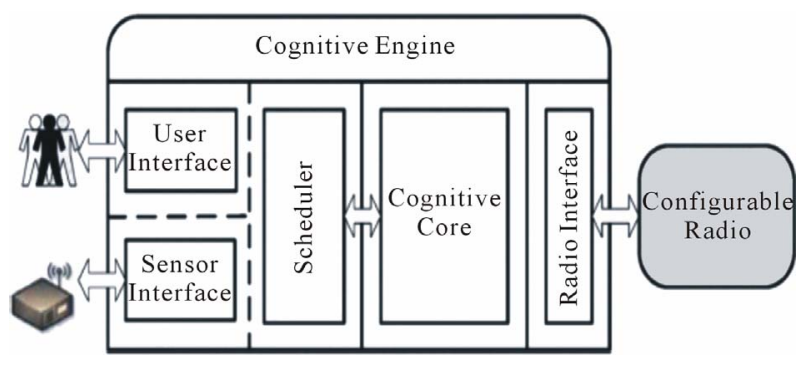

Figure 2. Cognitive engine. 
Recently soft computing techniques like ANN, genetic algorithm, Ant colony optimization are applied in the field of engineering for speech processing [39], telecommunications [40]. In Section 3 ANN is introduced briefly; in 4 we present applications of ANN for cognitive radio following the recent literature. In Section 5 the proposed ANN model for spectrum sensing keeping in view the channel capacity. Finally Section 6 concludes the paper with an overview of recent possible applications while outlining some of our ongoing research activities.

\subsection{Neural Networks for Spectrum Sensing}

Ref. [41] suggests Artificial neural networks for spectrum sensing. The disadvantages of Cyclostationary feature detection technique and Matched filter technique are overcome by the ANN model developed by combining the cyclostationary and the energy detection techniques.

The signal is assumed to be an AM signal in Additive White Gaussian Noise.

$$
\begin{aligned}
s(t) & =x(t)+w(t) \\
& =[A+\sqrt{2} \cos (2 \pi f t+\theta)] \cos \left(2 \pi f_{0} t+\theta_{0}\right)+w(t)
\end{aligned}
$$

The central frequency $f_{0}$ is taken $4 \mathrm{KHz}$. Sample signal frequency is assumed to be $1 \mathrm{~Hz}$ to $50 \mathrm{~Hz}$, Sample rate Fs is assumed to be $500 \mathrm{~Hz}$. The neural network is designed with four inputs: Energy of the signal and three cyclic spectrum values. These features are extracted for an AM signal with envelope $a(t)$ given by

$x(t)=a(t) \cos \left(2 \pi f_{0} t+\theta\right)$. The energy of the signal and cyclic spectrum values for cyclic frequencies $\alpha= \pm 2 f_{0}$ is taken as the input to the neural network. The network is trained for 300 iterations and the training goal is $10^{-5}$, learning rate is 0.05 and moment factor is 0.09 to predict SNR as shown in Figure 3.

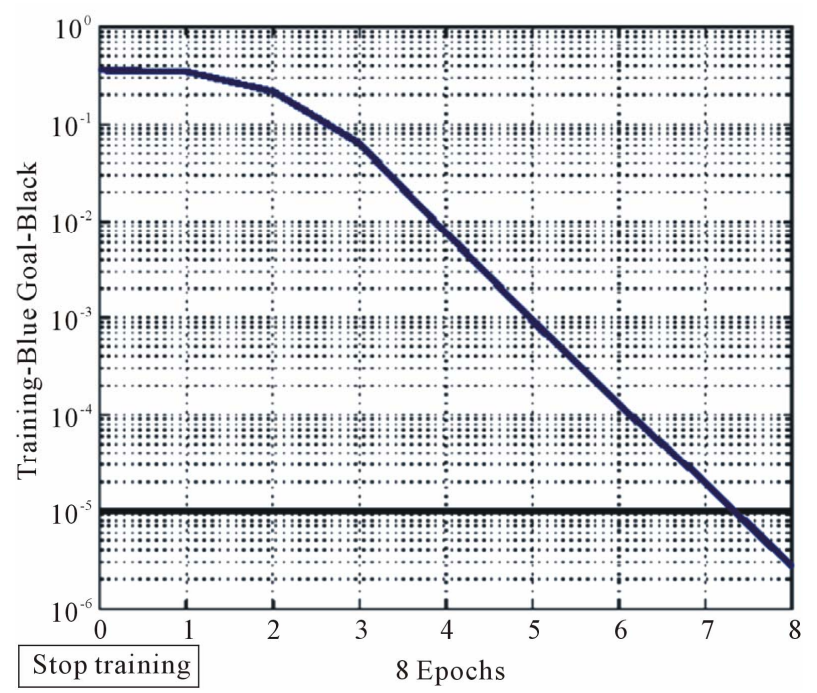

Figure 3. Training curve.
It is reported that the neural network acts efficiently for low SNR and the probability for false alarm is low.

\section{Artificial Neural Network}

Artificial Neural Networks (ANNs) are neuroscience inspired computational tools that are trained using inputoutput data to generate a desired mapping between input stimulus to the targeted output [42].

The artificial neuron is the unit model of the ANN structure which gets input from all neighboring neuron and gives an output depending on its synaptic weight and activation functions. Activation function like Tansig function, Sigmoid function are used.

The mathematical representation of the neuron model is given as

$$
\begin{gathered}
u_{k}=\sum_{j=1}^{m} w_{k j} x_{j} \\
y_{k}=\varphi\left(u_{k}+b_{k}\right)
\end{gathered}
$$

$x_{1}, x_{2}, x_{3}, x_{4}, \cdots, x_{m}$ are the inputs to the network, $w_{k 1}, w_{k 2}$, $w_{k 3}, w_{k 4}, \cdots, w_{k m}$ are the weights to the net where $u_{k}$ is the linear combiner and $b_{k}$ is the bias and $y_{k}$ is the output of the neuron.

The neurons are arranged in three layers the input layer, hidden layer and the output layer. Learning is accomplished by adjusting the weights till the difference between the desired output and the output of the network is negligible. The number of layers in the hidden layer and the number of neurons in each layers are changed on a hit and trial basis. Here a training algorithm is applied which helps the neural network to learn like back propagation algorithm, resilent back propagation, Levenberg algorithm, conjugate gradient, Quasi Newton algorithm etc. its variants are used. Once trained these models can be used directly to provide instant results for the purpose for which it has been developed [42].

The design and analysis of a Neural Network has the following phases:

- Generation of data;

- Training of the neural network;

- Testing.

The generation of the data is intended to decide the inputs and outputs which are to be trained. Training of the input parameters is done in such a way that the output of the neural network is same as the desired output. Hence this structure can be used further to predict the output in such an environment. The ANN has been used to make the cognitive radio to learn from the environment and take decision $[19,38]$.

\section{Problem Formulation}

In this paper we have tried to put up a new technique to 
identify spectrum holes. A neural network model which can predict the channel status whether occupied or unoccupied is designed for spectrum sensing. The channel information for the simulation here is the channel capacity, SNR, spectral efficiency of the channel scanned and distance " $d$ " between the primary base station and secondary base station/SU. Spectrum sensing implies the detection of white spaces. Here the proposed ANN model predicts the channel status as " 1 " for an occupied channel and " 0 " for unoccupied channel.

We consider a TV broadcast system with omnidirectional antenna and a reception system over an AWGN channel. The channel bandwidth is taken as $8 \mathrm{MHz}$ and TV transmit power $P_{t}$ as $50 \mathrm{KW}$ [44]. As we know the transmission range of a TV station is nearly $200 \mathrm{Km}$, a secondary user like a wi-fi, cellular system whose transmission range is less than that of the primary BS can use the unused TV band. The concept of cognitive radio is inculcated by allotting the vacant band to the SU. So a cognitive radio based scanning system with artificial intelligence is proposed. This CR based scanning system is placed within the coverage area of the primary TV base station (BS) at a distance " $d$ " from it as shown in Figure 4. The scanning system scans the channels licensed for the primary user PU and the SNR, bandwidth efficiency, channel capacity over the channel, and distance " $d$ " is given as input to the ANN model to predict the status of the channel. The distance " $d$ " is considered over a range of $100 \mathrm{~m}$ to $2000 \mathrm{~m}$. All the parameters given as input to the ANN model are an index to identify white spaces as explained further.

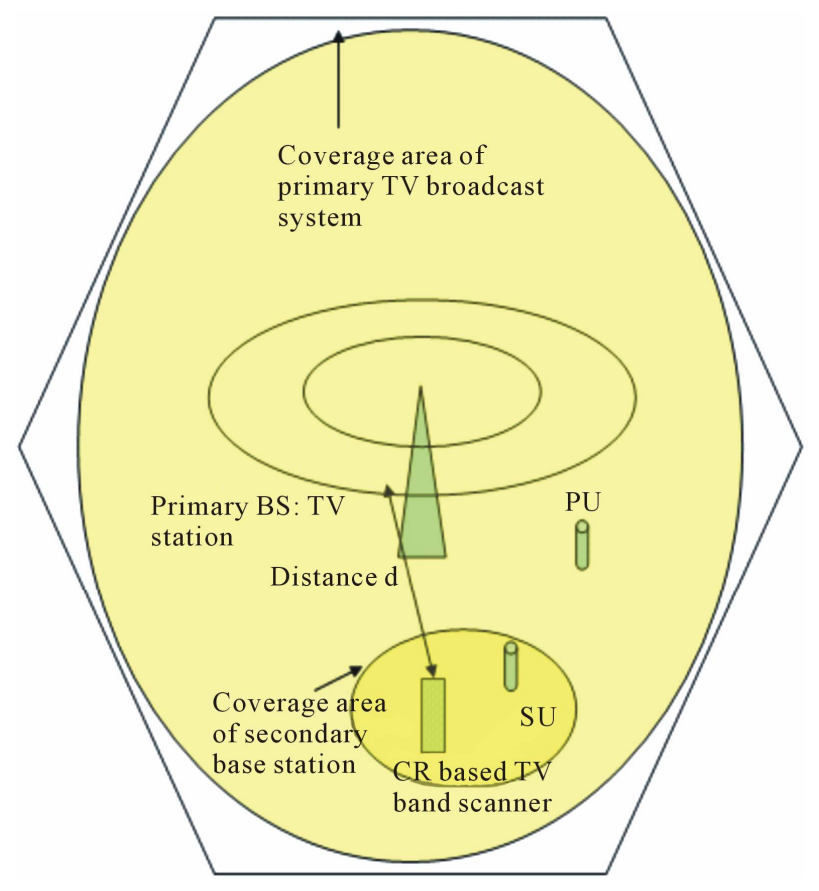

Figure 4. Proposed scenario.

\subsection{Signal to Noise Ratio}

We know that the power received $P_{r}$ at a receiver placed at a distance " $d$ " from the transmitter base station is dependent on the pathloss exponent " $n$ " and transmit power $P_{t}$, which is assumed to be $50 \mathrm{KW}$. So the power received at the receiver at a distance " $d$ " from the transmitter is given as [43]

$$
P_{r}(d)=P_{t}\left(d_{0} / d\right)^{n} \text { for } d_{0}=10 \mathrm{~m}
$$

Noise power spectral density $N_{0}$ is assumed as $10^{-9}$ $\mathrm{W} / \mathrm{Hz}$ and $B$ is $8 \mathrm{MHz}$ bandwidth and $d_{0}$ is the reference distance.

For $P_{t}$ equal to $50 \mathrm{KW}, n=3$ and distance $d=200 \mathrm{~m}$ SNR will be

$$
\mathrm{SNR}=P_{r}(d) /\left(N_{0} B\right)=28.9 \mathrm{~dB}
$$

At a distance $400 \mathrm{~m}$ the SNR will be $19.8 \mathrm{~dB}$. Hence SNR decreases with increasing distance $d$ but the channel has to be considered occupied. SNR if below a threshold level, than the channel can be considered as vacant. As per the FCC regulations the threshold regulation of actual TV receiver is $15.3 \mathrm{~dB}$ [44-46]. The ANN model has to detect the presence of analog and digital TV signal and voice signal. So the threshold level of SNR is taken as $-10 \mathrm{~dB}$.

\subsection{Channel Capacity}

The channel capacity $C$ is a very important parameter to analyze the channel status. The channel capacity here can be assumed as the transmission rate over the channel.

The channel capacity of a channel with bandwidth $B$ and SNR is given as [13]

$$
C=B \log _{2}(1+\mathrm{SNR})
$$

The channel capacity reduces as SNR reduces. If the channel is unoccupied then the noise power is high and the capacity will reduce. A low channel capacity indicates that the channel is vacant.

As per Shannon, channel capacity is also a measure of mutual information $I(X ; Y)$ and entropy $(H)$ over all possible inputs [13].

$$
C=\max _{p(x)} I(X ; Y)=[H(X)-H(Y \mid X)]
$$

where $X$ is the input and $Y$ is the output .

The channel capacity increases as the entropy increases [43].

If $\max _{p(x)} I(X ; Y) \approx 0$, then it means that the channel is not transmitting. Hence it is a decision making factor to declare a channel as occupied or unoccupied.

For this scenario channel capacity over a transmitting or occupied channel for distance " $d$ " equal to $200 \mathrm{~m}$ and for SNR equal to $28.9 \mathrm{~dB}$ is calculated as $C=76.8919$ Mbps. At this location if the channel capacity is found 
less than this value then it is a clear indication that the channel is unutilized. So the ANN model placed at $200 \mathrm{~m}$ from the transmitting base station, channel capacity is given as input along with the other parameters and is trained to predict the channel occupancy status. The SNR at a particular location is dependent on the distance between the primary TV base station and the geographical location at which the $\mathrm{CR}$ based scanning system is placed. We know that the SNR will reduce as the distance $d$ between the BS and CR based scanning system increases. Hence the channel capacity will reduce even though the channel is transmitting. So the threshold of channel capacity at which the channel is declared as unoccupied is dependent on the distance " $d$ ".

For distance " $d$ " being $200 \mathrm{~m}$ the channel capacity $C$ for an occupied TV channel is $76.8919 \mathrm{Mbps}$. At this distance for a channel with channel capacity less than this value can be declared as unoccupied. But for a scanning system placed at a distance $400 \mathrm{~m}$ from the transmitting base station the SNR received will be $19.8 \mathrm{~dB}$. The channel capacity of the occupied channel will be 52.9947 Mbps which is less than the channel capacity predicted at $200 \mathrm{~m}$ but the channel is occupied. Hence to identify spectrum hole at a particular geographical location in TV band a threshold level for SNR and the corresponding channel capacity has to be considered.

\subsection{Bandwidth Efficiency}

The bandwidth efficiency reflects how efficiently the allotted bandwidth is utilized. It is the throughput data rate per hertz in a given bandwidth [43].

$$
\eta=R / B \mathrm{bps} / \mathrm{Hz}
$$

where $R$ is the data rate in bit/sec and $B$ is the bandwidth allotted for the signal. Here $R$ is the channel capacity $C$, i.e. the transmission rate over the channel.

The bandwidth efficiency is calculated as [13]

$$
C / B=\log _{2}(1+\mathrm{SNR})
$$

where $C$ is channel capacity and $B$ is bandwidth. It is an index to declare the status of a channel.

Less bandwidth efficiency is due to no transmission over the channel. Spectral efficiency and mutual information if nearly zero or below a certain threshold then the channel can be considered as unoccupied. Hence the availability of the channel can be decided. A decision making threshold $\lambda$ has to be set for channel capacity above which the channel is considered as occupied and below it the channel is considered as unoccupied.

The method we are proposing may be applied both for WLAN in infrastructure mode as well as in ad-hoc mode. In case of infrastructure mode the access point must be equipped with $\mathrm{CR}$ based scanning system. The distance between the access point and the primary TV BS is fixed.
So the ANN model used here is for predicting the channel capacity for a fixed distance say $200 \mathrm{~m}$. Depending on the channel capacity predicted the channel status is decided. If the channel is unoccupied than the access point allots the channel to the SU in its coverage area.

Incase of ad-hoc mode all the mobile nodes have to be equipped with $\mathrm{CR}$ based scanning system. The input to ANN model is the distance between the mobile node and the primary base station and SNR over the scanned channel. The mobile node (SU) can scan for the unoccupied channel at every location. The channel status is decided based on the channel capacity predicted for a particular SNR and the distance " $d$ ".

In this paper we have trained the ANN model assuming that the SU can be an infrastructure system or an ad-hoc system. Hence the distance considered is over a range of $100 \mathrm{~m}$ to $2000 \mathrm{~m}$ from the primary TV transmitting station but placed within the coverage area of the primary transmission system.

\section{Proposed ANN Model}

The CR based scanning system we intend to develop must be equipped with a GPS system, SNR, channel capacity and spectral efficiency measuring equipment. The GPS is for detecting the current position of the secondary user and further calculated to determine the distance " $d$ " from the primary transmitting station. A TV channel is scanned and the SNR, channel capacity, spectral efficiency over it is determined, and all these values and distance " $d$ " are given as input to the ANN model to determine the channel's occupancy status.

The ANN model has to be developed so as to get accurate results. The efficiency of the neural network model depends on the parameters used for training. Database comprising the distance " $d$ ", SNR, channel capacity, spectral efficiency over a TV band and the channel status corresponding to these values is developed. The channel capacity is calculated for SNR ranging from -20 $\mathrm{dB}$ to $37 \mathrm{~dB}$ and for a bandwidth of $8 \mathrm{MHz}$. The distance " $d$ " is over a range of $100 \mathrm{~m}$ to $2000 \mathrm{~m}$. The threshold for SNR is taken as $-10 \mathrm{~dB}$. As per FCC $[45,46]$ minimum SNR over a transmitting channel has to be $15.3 \mathrm{~dB}$. Hence the channel capacity has to be $40.99 \mathrm{Mbps}$ and spectral efficiency has to be $5.12 \mathrm{bps} / \mathrm{Hz}$. This threshold level is set for proper reception of the signal.

But at level below this threshold the channel might be occupied with a faded signal. A TV band with SNR -10 $\mathrm{dB}$ is found to have a spectral efficiency $0.13 \mathrm{bps} / \mathrm{Hz}$ and channel capacity $1.1 \mathrm{Mbps}$ which is far below the standards. Therefore we consider this as the threshold for spectrum sensing.

The ANN model is designed and trained for different number of hidden layer and neurons in it and the results are compared. ANN model 1 is designed with 1 hidden 
layer having 5 neurons in it. ANN model 2 is designed with 1 hidden layer having 25 neurons in them. The ANN model 3 has 2 hidden layers with 10 and 5 neurons in them. All the ANN models are trained to a targeted accuracy of 0.00001 . Training is performed by using "trainrp", i.e. the resilent back-propagation algorithm. The sigmoid function "logsig" is used.

For any value of $n \log \operatorname{sig}(n)=1 /(1+\exp (-n))$. logsig is a transfer function that returns an output squashed to 0 or 1 [47]. Hence this transfer function is ideal if the output has to be either 1 or 0 . The performance of each ANN model is compared in the Figure 5. It is observed that the ANN model 3, as described in Table $\mathbf{1}$ has accuracy of mean absolute error 0.01676 and it yields relatively more accurate result as compared with ANN models 1 and 2.

From Table 2. it is observed that the channel status predicted by the neural network model is nearly same as the status as per theoretical calculations.

Hence the neural network model can be used for predicting the channel status. This model can be used as the AI core of the cognitive radio and predict the status of TV channel.

The channel status is analysed on the basis on $I_{\max }(X, Y)$ over the channel. As per Equation (10), channel capacity is the maximum mutual information over the channel. If the mutual information $I_{\max }(X, Y)$ of the channel is nearly equal to zero then the channel is considered as unoccupied.

From Figure 6 it is observed that the channel capacity over an occupied channel reduces as the SNR reduces. SNR reduces as the distance between the transmitting TV

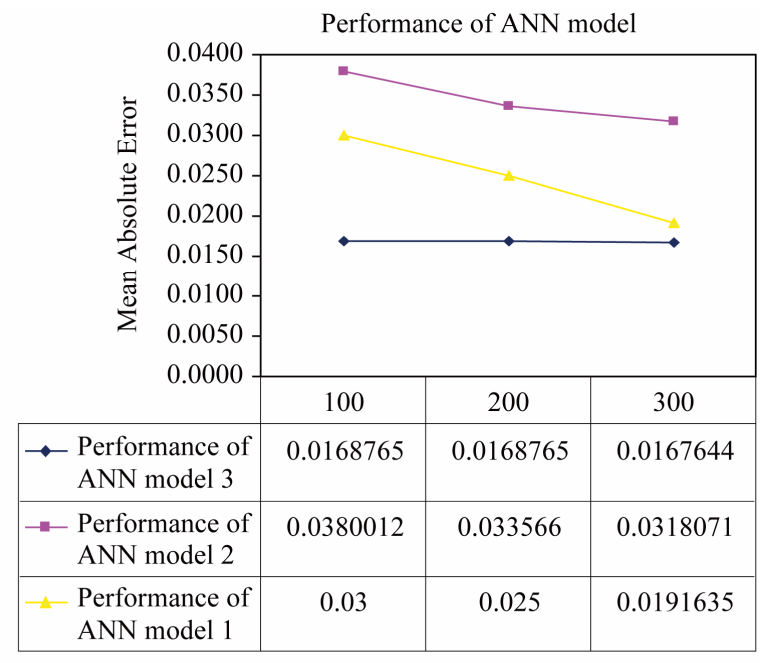

no:of iterations

Performance of ANN model $3-$ Performance of ANN model 2 Performance of ANN model 1

Figure 5. Performance comparison of different ANN models.
Table 1. Training parameters.

\begin{tabular}{cc}
\hline Parameters & Value \\
\hline Number of input neurons & 4 \\
Number of output neurons & 1 \\
Number of neuron in hidden layer 1 & 10 \\
Number of neuron in hidden layer 2 & 5 \\
Learning rate $(\eta)$ & 0.01 \\
Training tolerance & $1 \times 10^{-5}$ \\
Sigmoid function in hidden layer & logsig \\
Training Algorithm & Resilient Backpropagation \\
\hline
\end{tabular}

Table 2. Comparative study of ANN output and theoretical output.

\begin{tabular}{cccccc}
\hline $\begin{array}{c}\text { SNR in } \\
\mathrm{dB}\end{array}$ & $\begin{array}{c}\text { Channel } \\
\text { capacity } \\
\text { Mbps }\end{array}$ & $\begin{array}{c}\text { BW } \\
\text { efficiency } \\
\text { bps/Hz }\end{array}$ & d in m & $\begin{array}{c}\text { Channel } \\
\text { status } \\
\text { theoretical }\end{array}$ & $\begin{array}{c}\text { Channel status } \\
\text { predicted by } \\
\text { ANN model }\end{array}$ \\
\hline 28.9 & 76.8919 & 9.611 & 200 & 1 & 1 \\
-10 & 1.1 & 0.1375 & 300 & 0 & 0 \\
10 & 27.6755 & 3.4594 & 500 & 1 & 1 \\
3.5 & 13.7015 & 1.7127 & 1400 & 1 & 1 \\
7.9 & 22.8636 & 2.858 & 1000 & 1 & 1 \\
-20 & 0.1148 & 0.014 & 1100 & 0 & 0 \\
\hline
\end{tabular}

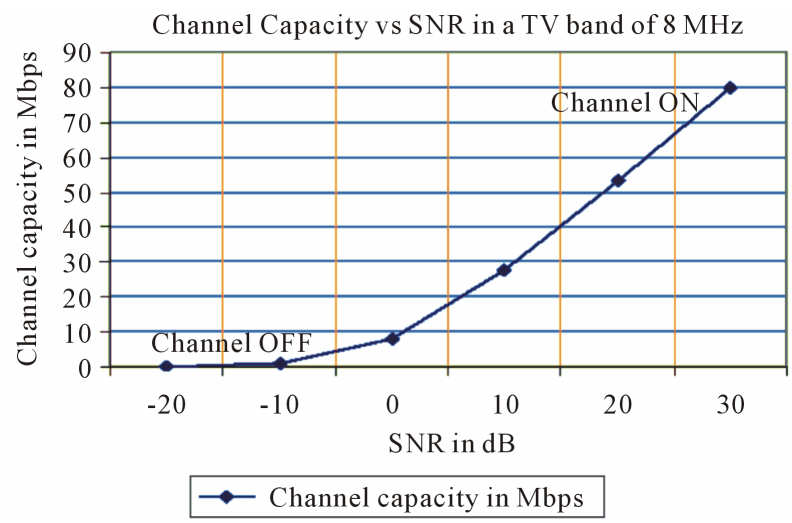

Figure 6. Channel capacity vs SNR in dB over TV channel.

station and $\mathrm{CR}$ based scanning system increases. At a distance " $d$ " of $500 \mathrm{~m}$ if a TV band of $8 \mathrm{MHz}$ is occupied, the SNR will be $19.8 \mathrm{~dB}$ the channel capacity will be $52.9947 \mathrm{Mbps}$ and bandwidth efficiency will be 6.624 bps/1 Hz. But if the spectral efficiency over this channel is below $0.1 \mathrm{bps} / \mathrm{Hz}$ and channel capacity is below 1.1 Mbps then channel at this geographical location can be assumed as unoccupied. As the channel capacity is reducing the bandwidth efficiency is nearly equal to zero. Hence a channel with less channel capacity has low 


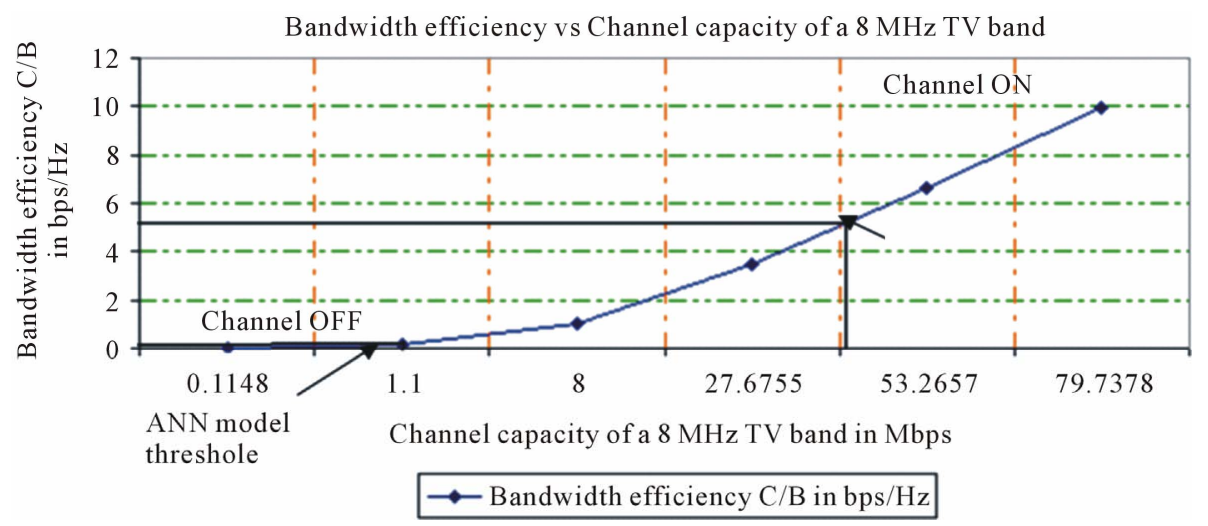

Figure 7. Simulation results on bandwidth efficiency and channel capacity over an occupied and unoccupied TV channel.

bandwidth efficiency i.e. the primary signal is absent and the channel can be declared as unoccupied.

From Figure 7 we observe that the spectral efficiency is reducing as the channel capacity is reducing. For a channel with channel capacity nearly equal to zero the spectral efficiency is also nearly equal to zero. Hence the channel can be declared as vacant or unoccupied. As per FCC in a TV band the channel capacity has to be 40.99 Mbps and spectral efficiency has to be $5.12 \mathrm{bps} / \mathrm{Hz}$ for effective reception. But as at this threshold the channel may contain the signal. The ANN model we designed has taken a threshold of $-10 \mathrm{~dB}$ at which the channel capacity is $1.1 \mathrm{Mbps}$ and spectral efficiency is $0.1375 \mathrm{bps} / \mathrm{Hz}$. So the ANN model is able to detect white spaces.

The ANN model designed tries to eliminate the disadvantages of the conventional spectrum sensing techniques like energy detection technique and autocorrelation technique. The energy detection technique is considered to flaw at low SNR and using the cyclostationary technique it is difficult to exploit the cyclostationary features in weak voice signals [48]. The ANN model designed can identify spectrum holes at SNR as low as -20 dB.

The CR based scanning system with ANN model predicts the state of the channel based on the channel's SNR, capacity and bandwidth efficiency, hence identify the white spaces. The optimized neural network model once trained need not be further trained and the optimized weights and bias can be extracted to design the CAD model. We further plan to consider factors affecting transmission of signal like interference and design a CAD model. This particular technique is most suitable for spectrum sensing and allocation in a particular coverage area of the primary TV station .

In this paper we have tried to put up a new technique to identify spectrum holes in TV band.

\section{Conclusion}

In this article we present a new ANN based model that is able to identify occupancy status of a given channel in the TV band spectrum with a view to subsequent utilisability following the CR concept. Essentially a prediction algorithm is proposed here for the identification of the spectrum hole that could be allotted to the aspiring SU. The occupancy is determined by examining some channel parameters, e.g. SNR, channel capacity, BW efficiency and distance $(d)$. The proposed algorithm indicates channel status occupancy in a quantized index form $\{0,1\}$ after appropriate training of the ANN engine. Simulation results match comparably with theoretically calculated ones are included. A brief and comprehensive overview of the recent ANN based literature for CR application has been added.

\section{REFERENCES}

[1] D. Cabric, S. M. Mishra and R. W. Brodersen, "Implementation Issues in Spectrum Sensing for Cognitive Radio," Proceedings of 38th Asilomar Conference on Signals, Systems, and Computers, Pacific Grove, 7-10 November 2004, pp. 772-776.

[2] I. F. Akyildiz, W. Y. Lee, M. C. Vuran and S. Mohanty, "Next Generation/Dynamic Spectrum Access/Cognitive Radio Wireless Networks: A Survey," International Journal of Computer and Telecommunications Networking, Vol. 50, No. 13, 2006, pp. 2127-2159.

[3] M. Gandetto and C. Regazzoni, "Spectrum Sensing: A Distributed Approach for Cognitive Terminals," IEEE Journal on Selected Areas in Communications, Vol. 25, No. 3 , 2007, pp. 546-557. doi:10.1109/JSAC.2007.070405

[4] M. Subhedar and G. Birajdar, "Spectrum Sensing Techniques in Cognitive Radio Networks: A Survey," Proceedings of International Journal of Next-Generation Networks, Vol. 3, No. 2, 2011, pp. 37-51.

[5] P. Kolodzy, et al., "Next Generation Communications: Kickoff Meeting," Proceedings of Defense Advanced Research Projects Agency, Arlington, 17 October 2001.

[6] M. McHenry, "Frequency Agile Spectrum Access Technologies," FCC Cognitive Radio Workshop, Washington DC, 19 May 2003. 
[7] G. Staple and K. Werbach, "The End of Spectrum Scarcity," IEEE Spectrum, Vol. 41, No. 3, 2004, pp. 48-52. doi:10.1109/MSPEC.2004.1270548

[8] A. Petrin and P. G. Steffes, "Analysis and Comparison of Spectrum Measurements Performed in Urban and Rural Areas to Determine the Total Amount of Spectrum Usage," Proceeding of 7th Annual ISART, Boulder, 1-3 March 2005.

[9] J. Mitola III and G. Q. Maguire Jr., "Cognitive Radio: Making Software Radios More Personal," IEEE Personal Communications Magazine, Vol. 6, No. 4, 1999, pp. 1318.

[10] X. Dong, Y. Li, C. Wu and Y. Cai, "A Learner Based on Neural Network for Cognitive Radio," 12th IEEE International Conference on Communication Technology, Nanjing, 11-14 November 2010, pp. 893-896.

[11] T. Yucek and H. Arslan, "A Survey of Spectrum Sensing Algorithms for Cognitive Radio Applications," IEEE Communications Surveys \& Tutorials, Vol. 11, No. 1, 2009, pp. 116-130. doi:10.1109/SURV.2009.090109

[12] M. Ghozzi, M. Dohler, F. Marx and J. Palicot, "Cognitive Radio Methods for the Detection of Free Bands," Comptes Rendus Physique, Vol. 7, No. 7, 2006, pp. 794-804. doi:10.1016/j.crhy.2006.07.009

[13] J. G. Proakis, "Digital Communications," McGraw-Hill Pub., New York, 2001.

[14] H. Urkowitz, "Energy Detection of Unknown Deterministic Signals," Proceedings of the IEEE, Vol. 55, No. 4, 1967, pp. 523-531. doi:10.1109/PROC.1967.5573

[15] F. F. Digham, M. S. Alouini and M. K. Simon, "On the Energy Detection of Unknown Signals over Fading Channels," IEEE Transactions on Communications, Vol. 55, No. 1, 2007, pp. 21-24. doi:10.1109/TCOMM.2006.887483

[16] I. A. Akbar, K. K. Bae, J.-S. Urn, C. M. Spooner and J. H. Reed, "Cyclostationary Approaches to Signal Detection and Classification in Cognitive Radio," 2nd IEEE International Symposium on New Frontiers in Dynamic Spectrum Access Networks (DySPAN 2007), Ireland, 17-20 April 2007, pp. 212-215. doi:10.1109/DYSPAN.2007.35

[17] S. Shankar, C. Cordeiro and K. Challapali, "Spectrum Agile Radios: Utilization and Sensing Architectures," Proceeding of IEEE International Symposium Ibid, Maryland, 8-11 November 2005, pp. 160-169.

[18] Y. J. Tang, Q. Y. Zhang and W. Lin, "Artificial Neural Network Based Spectrum Sensing Method for Cognitive Radio," 6th International Conference on Wireless Communications, Networking and Mobile, Chengdu, 23-25 September 2010, pp. 1-4.

[19] S. Pattanayak, P. Venkateswaran and R. Nandi, "Artificial Neural Network for Cognitive Radio: A Preliminery Survey," WiCOM 2012, Shanghai, 21-23 September 2012.

[20] F. Weidling, D. Datla, V. Petty, P. Krishnan and G. Minden, "A Framework for RF Spectrum Measurements and Analysis," IEEE International Symposium New Frontiers in Dynamic Spectrum Access Networks (DySPAN), Baltimore, 8-11 November 2005, pp. 573-576.

[21] D. C. Oh and Y.-H. Lee, "Energy Detection Based Spec- trum Sensing for Sensing Error Minimization in Cognitive Radio Networks," International Journal of Communication Networks and Information Security (IJCNIS), Vol. 1, No. 1, 2009, pp. 1-5.

[22] J. Lehtomaki, J. Vartiainen, M. Juntti and H. Saarnisaari, "Spectrumsensing with forward Methods," Proceedings of IEEE Military Communications Conference, Washington DC, 23-25 October 2006, pp. 1-7.

[23] J. Vartiainen, H. Sarvanko, J. Lehtomki, M. Juntti and M. Latva-aho, "Spectrum Sensing with LAD-Based Methods," Proceedings of 18th Annuals of IEEE International Personal, Indoor and Mobile Radio Communications Symposium (PIMRC), Athens, 3-7 September 2007, pp. 1-5.

[24] T. Yücek and H. Arslan, "Spectrum Characterization for Opportunistic Cognitive Radio Systems," Proceedings of IEEE Military Communications Conference, Washington DC, 23-25 October 2006, pp. 1-6.

[25] D. Cabric and R. W. Brodersen, "Physical Layer Design Issues Unique to Cognitive Radio Systems," Proceedings of 16th Annuals of IEEE International Personal, Indoor and Mobile Radio Communications Symposium (PIMRC), Vol. 2, Berlin, 11-14 September 2005, pp. 759-763.

[26] J. Proakis, "Digital Communications," McGraw-Hill, New York, 2001.

[27] Sahai and D. Cabric, "A Tutorial on Spectrum Sensing: Fundamental Limits and Practical Challenges," Proceeding of IEEE Symposium on New Frontiers in Dynamic Spectrum Access Networks (DySPAN), Baltimore, 8-11 November 2005.

[28] Y. Zeng and Y. C. Liang, "Spectrum-Sensing Algorithms for Cognitive Radio Based on Statistical Covariances," IEEE Transactions on Vehicular Technology, Vol. 58, No. 4, 2009, pp. 1804-1815. doi:10.1109/TVT.2008.2005267

[29] Y. Zeng and Y. Liang, "Covariance Based Signal Detections for Cognitive Radio," Proceeding of 6th IEEE Symposium on New Frontiers in Dynamic Spectrum Access Networks (DySPAN), Ireland, 17-20 April 2007, pp. 202207.

[30] A. Al-Dulaimi and L. Al-Saeed, "An Intelligent Scheme for First Run Cognitive Radios," 4th International Conference on Next Generation Mobile Applications, Services and Technologies, Jordan, 27-29 April 2010.

[31] J. H. Hauris, D. He, G. Michel and C. Ozbay, "Cognitive Radio and RF Communication Design Optimiasation Using Genetic Algorithm," MILCOM, Florida, 29-31 October 2007.

[32] G. Ganesan and Y. Li, "Agility Improvement through Cooperative Diversity in Cognitive Radio," Proceedings of IEEE Global Telecommunications Conference (GLOBECOM 2011), St. Louis, 28 November-2 December 2005, pp. 2505-2509.

[33] G. Ganesan, "Cooperative Spectrum Sensing in Cognitive Radio Networks," Proceedings of IEEE International Symposium on New Frontiers in Dynamic Spectrum Access Networks, Baltimore, 8-11 November 2005, pp. 137143.

[34] D. Cabric, A. Tkachenko and R. Brodersen, "Spectrum Sensing Measurements of Pilot, Energy, and Collaborative 
Detection," Proceedings of IEEE Military Communications Conference, Washington DC, 23-25 October 2006, pp. 1-7.

[35] P. Venkateswaran, S. Shaw, S. Pattanayak and R. Nandi, "Cognitive Radio Ad-Hoc Networks: Some New Results on Multichannel Hidden Terminal Problem," Communications and Network, Vol. 4 No. 4, 2012, pp. 342-348. doi:10.4236/cn.2012.44039

[36] Q. Zhao and B. Sadler, "A Survey of Dynamic Spectrum Access," IEEE Signal Processing Magazine, Vol. 24, No. 3, 2007, pp. 79-89. doi:10.1109/MSP.2007.361604

[37] B. Wang and K. J. R. Liu, "Advances in Cognitive Radio Networks: A Survery," IEEE Journal of Selected Topics in Signal Processing, Vol. 5, No. 1, 2011, pp. 5-23.

[38] X. Dong, Y. Li, C. Wu and Y. Cai, "A Learner Based on Neural Network for Cognitive Radio," 12th IEEE International Conference on Communication Technology (ICCT), Nanjing, 11-14 November 2010, pp. 893-896.

[39] S. Dhanjal, "Artificial Neural Networks in Speech Processing: Problems and Challenges," IEEE Pacific Rim Conference on Communications, Computers and Signal Processing, Victoria, 26-28 August 2001, pp. 510-513.

[40] P. Giribone, R. Revetria, M. Antonetti and R. Tabolacci, "Use of Artificial Neural Networks as Support for Energy Saving Procedures in Telecommunications," Telecommunications Energy Conference (INTELEC), Phoenix, 10-14
September 2000, pp. 159-162.

[41] Y. J. Tang, Q. Y. Zhang and W. Lin, “Artificial Neural Network Based Spectrum Sensing Method for Cognitive Radio," 6th International Conference on Wireless Communications, Networking and Mobile Computing (WiCOM), Chengdu, 23-25 September 2010, pp. 1-4.

[42] S. Haykin, "Neural Networks: A Comprehensive Foundation," Prentice-Hall, Upper Saddle River, 1999.

[43] Goldsmith, "Wireless Communication," Cambridge Press, Cambridge, 2005.

[44] J. Sachs, I. Maric and A. Goldsmith, "Cognitive Cellular Systems within the TV Spectrum," Proceedings of IEEE DySPAN, Stanford, 6 April 2010, pp. 1-12.

[45] S. R. Martin, "Test of ATSC 8-VSB Reception Performance of Consumer Digital Television Receivers," 2005 FCC/OET TR. 05-1017, Washington DC, 2 November 2005.

[46] Bendov, "Transmitter SNR for Maximim Coverage," IEEE Transaction on Broadcasting, Vol. 5, No. 4, 2008, pp. 784-785.

[47] http://www.mathworks.com/help/nnet/ref/logsig.htm

[48] S. J. Shellhammer, A. K. Sadek and W. Zhang, "Tehenical Challenges for Cognitive Radio in the TV White Space Spectrum", Proceedings of Information Theory and Applications Workshop, California, 2009, pp. 323-333. 